ISSN $1515-7326$, n. $^{\circ} 18,2 \mid 2016$, pp. 59 a 79

\title{
A propósito de umbrales, prototipos y usos de los estándares prueba
}

\author{
On Thresholds, Prototypes and Uses \\ of the Standard of Proof
}

Raymundo Gama*

Recepción y evaluación de propuesta: 15/04/2016

Aceptación: 15/7/2016

Recepción y aceptación final: 4/7/2017

Resumen: En este trabajo se plantean algunos comentarios a "Los usos de los estándares de prueba" de Rodrigo Coloma. El análisis del término «estándar» en el lenguaje ordinario y en el lenguaje jurídico puede arrojar luz sobre la noción de estándar de prueba. Sin embargo, resulta problemática la propuesta de entender el estándar de prueba en el derecho como un umbral de carácter cuantitativo o como un prototipo de carácter cualitativo que permite establecer relaciones de semejanza para considerar un hecho como probado. Por otra parte, es relevante la tarea de examinar los usos de los estándares de prueba, pero encuentro algunos problemas en la formulación de algunos de los usos que detecta Coloma. Finalmente, se plantea que la discusión sobre la posibilidad de formular un estándar de prueba realmente objetivo suele presentarse en términos dilemáticos: o bien el estándar de prueba es completamente objetivo o bien no es un estándar en absoluto. Frente a esta postura se destaca la tesis de Susan Haack en el sentido de que el estándar de prueba es en parte psicológico y en parte epistemológico. Palabras clave: estándares, umbral, prototipo, estándar de prueba, probabilismo jurídico

* ITAM. Departamento de Derecho. Ciudad de México, México. 


\begin{abstract}
This paper examines Rodrigo Coloma's "The uses of the standards of proof". The analysis of the word «standard» in both ordinary and legal language may shed light on the concept of standard of proof. However, Coloma's distinction between a quantitative threshold or a qualitative prototype is problematic. I also find problematic some of the uses of the standards of proof formulated by Coloma. Legal scholars traditionally argue that a subjective standard is not a standard at all. Against this view the paper stresses Susan Haack's view that the legal standard of proof is in part psychological and in part epistemological.

Keywords: Standards, thresholds, prototypes, standard of proof, legal probabilism
\end{abstract}

\title{
I. Introducción
}

En su artículo "Los usos de los estándares de prueba", Coloma se enfoca en el estándar de prueba, uno de los temas que más discusión ha suscitado entre quienes se ocupan del tema de la prueba ${ }^{1}$. En dicho trabajo, Coloma persigue dos propósitos. Por un lado, propone llevar a cabo una precisión conceptual de la noción de estándar de prueba en la que, junto al habitual sentido de «estándar» como umbral, identifica un segundo sentido de «estándar» como prototipo. A su modo de ver, estos dos sentidos servirían para entender mejor el estándar de prueba y los problemas prácticos que involucra su utilización en el derecho.

1 Véase, entre otros, Laudan, L., "Por qué un estándar de prueba subjetivo y ambiguo no es un estándar", Doxa, 28, 2005, pp. 95-13; Gascón, M., "Sobre la posibilidad de formular estándares de prueba objetivos", Doxa, 28, 2005, pp. 127-139; Igartua, J., "Prolongaciones a partir de Laudan", Doxa, 28, 2005, pp. 1412-1150; Fernández, M., "La valoración de la prueba y el estándar de duda razonable", Cuadernos Electrónicos de Filosofia del Derecho, 2007, 15; Ferrer, J., La valoración racional de la prueba, MadridBarcelona: Marcial Pons, 2005; Bayón, J. C., "Epistemología, moral y prueba de los Hechos: hacia un enfoque no benthamiano", Analisi e Diritto, 2008, pp. 15-34.; Accatino, D. "Certezas, dudas y propuestas en torno al estándar de la prueba penal", Revista de Derecho de la Pontifica Universidad Católica de Valparaíso, 2011, pp. 483-511 e Igartua, J., "Tomando en serio la duda razonable: enseñanzas de un controvertido caso judicial", en Derecho procesal: dilemas sobre la verdad en el proceso judicial, 2014, Medellín: Editorial Universidad Pontificia Bolivariana, pp. 135-162. 
A propósito de umbrales, prototipos y usos de los estándares prueba

Por otra parte, Coloma detecta una visión reduccionista en el análisis tradicional del estándar de prueba que se limita a considerarlo como un mecanismo para la distribución de errores ${ }^{2}$. A su modo de ver, el estándar de prueba tendría otros usos o funciones que sería importante identificar y examinar.

En este trabajo formularé algunas observaciones a las tesis planteadas por Coloma. Para ello presentaré brevemente las tesis que sostiene el autor y formularé algunos comentarios al respecto. Siguiendo una estructura análoga a la de Coloma, me ocuparé en primer lugar de su tesis sobre los dos sentidos de la noción de estándares de prueba. Posteriormente, me centraré en sus planteamientos sobre los usos de los estándares de prueba. Al igual que Coloma, considero que el análisis de la noción de estándar en el lenguaje ordinario y en el derecho puede arrojar luz sobre la noción de estándar de prueba. Sin embargo, soy escéptico respecto de su propuesta de entender el estándar de prueba como un umbral de carácter cuantitativo o como un prototipo de carácter cualitativo. Por otra parte, al igual que Coloma, me parece relevante examinar los usos de los estándares de prueba. No obstante, la formulación que lleva a cabo Coloma de los usos del estándar de prueba plantea algunos problemas.

\section{La noción de estándar en el lenguaje ordinario}

El trabajo de Coloma parte de la base de que el análisis del término «estándar» en el lenguaje ordinario puede contribuir a clarificar la noción de estándar de prueba en el derecho. Siguiendo esta metodología, Coloma plantea que habría dos sentidos de «estándar» que resultarían relevantes para este propósito.

En un primer sentido, Coloma señala que «estándar» es entendido como un umbral que establece el valor mínimo de pertenencia a una

2 Cfr. Coloma, p. 26: "En lo que sigue, sugeriré que los estándares de prueba son más operativos de lo que prima facie se les hace aparecer y que el juicio adverso que sobre ellos recae es consecuencia de una visión reduccionista de sus distintos usos". 
determinada categoría. De acuerdo con el autor, en esta primera acepción el estándar tendría un carácter "puramente cuantitativo", por lo que si se iguala o supera la cifra indicada en el estándar se resuelve positivamente la pertenencia de un caso a una determinada categoría ${ }^{3}$. Por el contrario, cualquier ejemplar que se ubique por debajo del indicador establecido por el estándar quedaría excluido de una determinada categoría.

En un segundo sentido, Coloma afirma que «estándar» puede ser definido como un "prototipo que reduce los problemas de extensión de una categoría"4. En esta segunda acepción, la noción de estándar supone que se cuenta con un ejemplar o prototipo que indudablemente se considera que pertenece a una determinada categoría. De este modo, la pertenencia o exclusión a una determinada categoría se resuelve mediante un análisis de carácter cualitativo en el que se examinan las semejanzas o diferencias del caso dudoso con el prototipo.

$\mathrm{Al}$ respecto, Coloma parte de un presupuesto metodológico acertado al advertir que el análisis de la noción de «estándar» en el lenguaje ordinario puede arrojar luz sobre la noción de «estándar de prueba» en el derecho. En efecto, es posible que entre el uso de esa expresión en uno y otro contexto haya algún tipo de conexión que resulte ilustrativa y que permita comprender mejor el estándar de prueba en el derecho. Sin embargo, conviene advertir que del hecho de que se utilice la misma expresión en dos contextos no se sigue que en cada uno de ellos se utilice dicha expresión con el mismo significado, ni que un sentido que esté presente en un determinado contexto pueda trasladarse automáticamente a otro. Una importación de este tipo no siempre es viable y no está garantizado que conduzca, en todos los casos, a buenos resultados ${ }^{5}$.

Ciertamente, la noción de estándar en el lenguaje ordinario puede estar relacionada de algún modo con los distintos estándares utilizados en el derecho, pero esto es precisamente lo que habría que mostrar. Habría que analizar de qué modo y en qué sentido podría haber

3 Vid. Coloma, Los usos de los estándares de prueba: entre umbrales y prototipos, artículo correspondiente a esta Discusión.

4 Vid. Coloma, op. cit.

5 Para una crítica en este sentido Vid. Allen, R. J., "Burdens of proof", Law, Probability and Risk, 2015, 3 (3-4), pp. 195-219. 
A propósito de umbrales, prototipos y usos de los estándares prueba

una relación o relaciones entre las nociones de estándar en el lenguaje ordinario, la noción de estándar en el lenguaje jurídico y por último la noción de estándar de prueba en el derecho.

Pero esta no es la vía que toma Coloma. Él identifica dos sentidos de «estándar» en el lenguaje ordinario para trasladarlos directamente a la noción de estándar de prueba en el derecho. Esta estrategia resulta problemática desde el inicio. Para mostrar por qué, es conveniente examinar brevemente y sin ningún ánimo de exhaustividad algunos de los significados más relevantes de los términos «estándar», «umbral» y «prototipo» en el lenguaje ordinario.

En relación con el término «estándar» en español es posible advertir un primer sentido que alude a lo que se considera que es común, habitual, usual o corriente ${ }^{6}$. Por ejemplo, cuando hablamos de la altura estándar de una mesa o un techo, del tamaño estándar de un tornillo o de la medida estándar de un póster o cartel. En un segundo sentido, «estándar» alude a algo que sirve de patrón, modelo o punto de referencia para medir o valorar cosas. En este segundo sentido se hace referencia, por ejemplo, al estándar de calidad del aire, al estándar de calidad de vida o al estándar de criminalidad. En relación con este segundo sentido, conviene advertir que los parámetros o indicadores utilizados pueden ser numéricos, pero no necesariamente es así en todos los casos. Para evaluar el estándar de vida, por ejemplo, se pueden tomar en cuenta factores numéricos como el nivel de ingreso y de gastos, pero también factores no numéricos como el nivel de bienestar personal y físico, el nivel de desarrollo personal, el grado del goce de derechos, la capacidad de autodeterminación, etc.

A su vez, el término «standard» en inglés es definido como "Un nivel de cualidad o de realización" y en el sentido de "Algo utilizado como medida, norma o modelo en comparaciones evaluativas"7.

6 Diccionario Anaya de la Lengua, Madrid, Anaya, 1991; Diccionario de la Lengua Española, España, La Academia, 2001.

7 «Standard», Oxford English Dictionary Online, junio 2017, Oxford University Press. "1. A level of quality or attainment" y " 2 . Something used as a measure, norm, or model in comparative evaluations". 
Por su parte, entre los significados del término «umbral» se encuentran los siguientes: 1) "la parte inferior en el vano de una puerta", 2) "entrada a cualquier cosa" y 3) "valor mínimo de un agente físico o estímulo capaz de producir un efecto". Finalmente, el término "prototipo» es definido como el primer molde o modelo y como el "mejor ejemplar de una cualidad o vicio".

Esta descripción de algunos de los significados de las expresiones anteriores no pretende ser exhaustiva, pero al menos permite poner de relieve que no hay una relación clara entre las nociones de estándar y umbral, por un lado, y las nociones de estándar y prototipo, por el otro, o al menos no hay una relación en los términos que presenta Coloma.

Coloma pone como ejemplo del uso de la noción de estándar en el sentido de umbral el siguiente enunciado: "De acuerdo con los estándares modernos, la casa es demasiado pequeña". En palabras del autor:

de un estándar se espera la elección entre una serie de variables determinantes de lo que es una casa pequeña o no pequeña (por ejemplo, metros cuadrados construidos, cantidad de piezas, etc.) y la estipulación de un nivel básico de satisfacción (por ejemplo, $60 \mathrm{~m}^{2}$ construidos, dos piezas, etc.) $)^{8}$.

Sin embargo, a diferencia de lo que señala Coloma, me parece que en este caso se está aludiendo más bien a la noción de estándar en el sentido de ciertos criterios o parámetros común o usualmente aceptados de lo que se considera una vivienda pequeña (o no), que a un valor mínimo de carácter numérico que indica la pertenencia a una determinada categoría.

Por otro lado, Coloma pone como ejemplo del uso de la noción de estándar en el sentido de prototipo el siguiente enunciado: "Este libro es el estándar respecto del cual todos los demás deberán ser juzgados". Este segundo ejemplo me parece menos problemático, pero en este caso la noción de estándar sería más próxima a las nociones de modelo o patrón que a la noción de prototipo. 
A propósito de umbrales, prototipos y usos de los estándares prueba

Hay, con todo, una razón adicional por la que me parece problemática la tesis de Coloma. Del hecho de que la noción de estándar de prueba en el derecho sea entendida y definida en términos de un umbral no se sigue que el término estándar en el lenguaje ordinario esté asociado también con la noción de umbral, ni mucho menos que este tenga un carácter cuantitativo9. Y a su vez, del hecho de que el término estándar sea entendido como modelo o patrón, o inclusive como prototipo como sugiere Coloma, no se sigue que el estándar de prueba pueda ser entendido como prototipo de carácter cualitativo.

Habiendo planteado estas primeras observaciones, sigamos examinando las tesis de Coloma.

\section{La noción de estándar en el lenguaje jurídico}

Tras examinar el término estándar en el lenguaje ordinario, Rodrigo Coloma dedica un apartado a examinar los estándares en el derecho. La pregunta específica que se plantea es "¿qué implica la partícula «estándar» en los contextos jurídicos?”. Coloma señala que a diferencia de lo que ocurre en el Common Law, en el derecho continental se ha reflexionado poco sobre los estándares en el derecho. A su juicio, esta falta de atención puede atribuirse al hecho de que hasta hace algunos años el término «estándar» estaba prácticamente ausente en el discurso de los jueces y abogados de nuestra tradición jurídica.

Adicionalmente, Coloma identifica cinco razones que impulsan el uso de estándares en distintos escenarios jurídicos: 1) los estándares se utilizan en contextos de toma de decisión; 2) la función de los estándares es resolver conflictos de pertenencia a una determinada

9 Un ejemplo de un umbral que no se presenta necesariamente en términos cuantitativos es el denominado umbral del dolor, el cual es entendido como la intensidad mínima de un estímulo que genera la sensación de dolor o la capacidad que tenemos los seres humanos de soportar la sensación de dolor. En algunos contextos (como en el de las pruebas aplicadas a neonatos, por ejemplo) se habla de "medir el dolor", pero más que medirlo, como se mediría el tamaño de un techo, se estaría evaluando el dolor a partir de ciertos signos o de la práctica de ciertas pruebas o técnicas utilizadas. 
categoría; 3) los estándares son útiles para resolver problemas de pertenencia de casos que se encuentran en la zona de penumbra, siendo poco útiles respecto de casos que se encuentran en la zona de claridad; 4) los estándares contribuyen a la conservación de un nivel razonable de complejidad de los casos que se evalúan y 5) los estándares consideran variables contextuales ${ }^{10}$.

En relación con este punto, el análisis que lleva a cabo Coloma se enfrenta a dos dificultades. En primer lugar, en el trabajo de Coloma se puede advertir una ambigüedad en el tratamiento de la noción de estándar en el lenguaje jurídico. En segundo lugar, Coloma parece limitar la función de los estándares a la de una herramienta que nos permite resolver problemas de pertenencia a una determinada categoría.

Respecto del primer punto, resulta pacífico que en el derecho recurrimos frecuentemente a estándares y que se trata de una noción que está presente en el lenguaje jurídico. Sin embargo, en el derecho hablamos de estándares en distintos tipos de situaciones y contextos. En algunas ocasiones hablamos de estándar en un sentido análogo al de principio, en tanto enunciados jurídicos distintos de las reglas, como cuando se contrapone la prohibición de circular a una velocidad excesiva con la prohibición de circular a más de ciento veinte kilómetros por hora ${ }^{11}$. En otras ocasiones, hablamos de estándares para aludir a ciertos parámetros jurídicos. Tal es el caso, por ejemplo, del estándar de "real malicia" o "malicia efectiva" utilizado para examinar si ciertas expresiones o discursos pueden considerarse difamatorios ${ }^{12}$. También se utiliza la noción de estándar en el sentido de un test o instrumento

10 Vid. Coloma, op. cit.

${ }^{11}$ No puedo ocuparme en este punto de la distinción entre reglas y estándares ni de la discusión relativa a si los estándares son un enunciado jurídico distinto de los principios o un tipo de principio. Simplemente quiero llamar la atención de que en algunos contextos, especialmente en el common law, se suele hablar de «estándares» como enunciados distintos de las reglas. Al respecto, Vid. Kaplow, L. "Rules versus standards: an economic analysis", 1992, 43, Duke Law Journal y Schauer, F. "The convergence of rules and standards", New Zealand Law Review, 2003, p. 3.

12 El estándar de malicia efectiva plantea que la imposición de sanciones civiles derivada de la emisión de opiniones, ideas o juicios está condicionada a que la información difundida se haya emitido con intención de dañar o a sabiendas de que la información era falsa. 
A propósito de umbrales, prototipos y usos de los estándares prueba

para controlar la regularidad de las normas y que varía en función de su intensidad; tal es el caso, por ejemplo, de los estándares de escrutinio estricto (o intenso) o intermedio. $Y$ en fin, entre estos y muchos otros estándares que se emplean en el derecho se encontrarían los estándares de prueba.

No pretendo ofrecer una presentación exhaustiva de los distintos contextos en los que operan estándares en el derecho. Tampoco pretendo sostener que la noción de estándar no esté (o pueda estar) relacionada de algún modo con alguna noción de estándar en el derecho. Al contrario, mi impresión es que la noción de estándar de prueba puede estar conectada de algún modo con la noción de estándar en el derecho y que el análisis de esta última puede arrojar luz sobre la primera. Sin embargo, me parece que habría que explorar más esta eventual conexión y mostrar los resultados a los que conduce. El planteamiento de Coloma puede ser entendido como una invitación más que bienvenida para reflexionar más sobre este punto.

Respecto del segundo punto, Coloma subraya en distintos momentos que los estándares (dentro y fuera del derecho) sirven para dirimir conflictos sobre la pertenencia de un caso dudoso a una determinada categoría. Sin embargo, al plantear de este modo la función de los estándares se corre el riesgo de perder de vista los distintos usos que se hacen de los estándares en los diferentes contextos en los que operan.

Coloma podría objetar que en muchas de estas situaciones nos encontramos precisamente con una discusión acerca de la pertenencia de un caso a una determinada categoría. Por ejemplo, si cierta información puede ser considerada calumniosa, si una norma es constitucional o no, si un hecho puede considerarse como probado. Pero, nuevamente, me parece que de este modo se obscurece la función que pueden llegar a desempeñar los estándares en distintos contextos, lo cual contrasta por cierto con su objetivo de identificar los potenciales usos de los estándares de prueba. 


\section{La noción de estándar de prueba}

En el apartado relativo a qué es un estándar de prueba Coloma plantea que la decisión de considerar un hecho como probado depende de tres elementos ${ }^{13}$ : i) una proposición (por ejemplo, "Dagoberto privó de la vida a Oscar Emmanuel"; "Juan sabía que se transportaba droga en el interior del vehículo en el que viajaba"), ii) una actitud, que en el trabajo de Coloma se limita a una actitud de aceptación o rechazo y iii) un conjunto de razones que sustentan la actitud adoptada. Coloma señala que el contenido de la proposición que establece los hechos a probar está determinado por las normas jurídicas. En este contexto, Coloma indica que el estándar de prueba determina si ciertos casos dudosos deben o no ser situados en la categoría de hechos probados. Para este fin, el estándar de prueba tiene como cometido "dar indicaciones suficientes acerca de las condiciones de las cuales se hace depender la actitud 'hechos probados' sí, y su opuesta, 'hechos probados' no".

Coloma proyecta a los estándares de prueba los sentidos de estándar como umbral y como prototipo. Su tesis es que el estándar de prueba puede ser entendido ya sea como umbral o como prototipo. El ejemplo paradigmático que ilustraría a su juicio la noción de estándar como umbral sería el estándar de "preponderancia de prueba» (PP). Siguiendo la noción de umbral que había planteado al analizar la noción de estándar en el lenguaje ordinario, Coloma sostiene el estándar de prueba en el sentido de umbral supone que se pueden construir escalas de medición y que el cálculo probabilístico resultaría adecuado para ese propósito ${ }^{14}$.

Respecto del estándar de prueba como prototipo, Coloma sostiene que su utilización supone contar con un caso no dudoso que permita establecer criterios de semejanza para determinar si entra o no en la categoría de hecho probado. El estándar que a su juicio ilustraría la noción de estándar de prueba como prototipo sería el estándar de pruebas «claras y convincentes» (CC). Finalmente, plantea que el estándar 
A propósito de umbrales, prototipos y usos de los estándares prueba

de prueba «más allá de toda duda razonable» (MADR) puede ser interpretado tanto como umbral y como prototipo.

Tras esta apretada síntesis de las tesis de Coloma, me gustaría formular dos observaciones.

(i) La primera observación tiene que ver con la reconstrucción del estándar de prueba como un umbral de carácter cuantitativo que presupone la posibilidad de establecer cuantitativamente la suficiencia de prueba. A pesar de que Coloma advierte que el empleo del cálculo numérico de probabilidades en el estándar de prueba plantea algunas dificultades, acaba aceptando la noción de estándar como un umbral que establece un quantum determinado de probabilidad. En relación con este punto, me parece que no hay margen para titubear al respecto pues nos encontramos ante una disyuntiva que no admite matices. Como plantea Juan Carlos Bayón: "o bien se sostiene que como resultado del razonamiento probatorio se puede expresar numéricamente el grado de probabilidad de que una hipótesis sobre los hechos sea verdadera, o bien se mantiene que tal cosa no es posible"15. La primera opción de la disyuntiva ha sido prácticamente descartada por la mayor parte de la comunidad jurídica, incluyendo a los autores que en nuestra tradición se han ocupado de la prueba. Todos estos autores coinciden en que el cálculo de probabilidades no es adecuado para el estándar de prueba ${ }^{16}$.

Susan Haack ha planteado también este punto cuando sostiene que "no se debe asumir solamente porque aparezcan palabras como 'probable', 'probablemente' y 'probabilidad' en contextos jurídicos, que se trata de probabilidades matemáticas, en lugar de epistemológicas". Como sostiene Haack, el cálculo matemático de probabilidades resulta adecuado en aquellos ámbitos en los que resulta aplicable. El establecimiento del grado de suficiencia del estándar de prueba en el derecho no sería uno de ellos toda vez que "no es factible asignar números pre-

15 Bayón, J. C., op. cit.

16 Gascón, M., op. cit.; Ferrer, J., op. cit.; Bayón, J. C. op. cit.; González Lagier, D., "Presunción de inocencia, verdad y objetividad", en García Amado, J. A. y Bonorino, P (coords.), Prueba y razonamiento probatorio, Granada, 2014, pp. 85-117. 
cisos a los grados de prueba y tampoco sería necesariamente deseable hacerlo, incluso si esto fuese posible"17.

Habiendo dejado a un lado la posibilidad de formular el estándar de prueba en términos numéricos, la discusión se ha centrado en establecer si es posible formular un estándar de prueba objetivo ${ }^{18}$. Hay quienes se decantan por emprender esfuerzos para formular un estándar de prueba de este tipo (por ejemplo, Larry Laudan, Ronald Allen, Michael Pardo y Jordi Ferrer $)^{19}$ y, por el contrario, quienes sostienen que tal empresa presenta varias dificultades (Mercedes Fernández, Juan Carlos Bayón y Daniel González Lagier). Frente a tales dificultades, los partidarios de esta última alternativa han planteado un cambio de enfoque en el que, según argumentan, debemos descartar por inviable el intento de formular un estándar de prueba completamente satisfactorio, debiendo optar más bien por el establecimiento de reglas sobre la prueba que incidan en la admisibilidad de los medios de prueba, en su valoración o en la carga de la prueba ${ }^{20}$.

${ }^{17}$ Haack, S., "El probabilismo jurídico. Una disensión epistemológica”, en Vázquez, C. (ed.), Estándares de prueba y prueba científica. Ensayos de epistemología jurídica, Madrid: Marcial Pons, 2013, pp. 65 y ss.; también publicado en Haack, S., Evidence matters. Science, Proof and Truth in the Law, New York: Cambridge, 2014, p. 58.

${ }^{18}$ De acuerdo con Bayón, serían cuatro los requisitos que tendría que cumplir un estándar de prueba de estas características: 1 . no debe tratarse de un estándar subjetivo, 2 . el estándar de prueba tiene que estar formulado en términos que permitan un control intersubjetivo, 3. "su formulación debe ser tal que de su aplicación correcta resulte exactamente la distribución del riesgo que se reputa justificada" y 4. de su aplicación debe resultar la distribución del riesgo establecida". Vid. Bayón, J. C., op. cit., quien a su vez los retoma con alguna reelaboración de Laudan, L., Verdad, error y proceso penal, trad. Carmen Vázquez y Edgar Aguilera, Marcial Pons, 2013, p. 126 y ss. y de Ferrer, J., La valoración racional de la prueba, 2007, p. 146.

19 Allen, R., "Los estándares de prueba y los límites del análisis jurídico", en Vázquez C. (ed.), Estándares de prueba y prueba científica. Ensayos de epistemología jurídica, Madrid: Marcial Pons, 2013, p. 65 y ss.; Pardo, M., "Estándares de prueba y teoría de la prueba", en Vázquez C. (ed.), Estándares de prueba y prueba científica. Ensayos de epistemología jurídica, Madrid: Marcial Pons, 2013, p. 99 y ss. y Ferrer, J., op. cit.

${ }^{20}$ Mercedes Fernández considera que el estándar de prueba «más allá de toda duda razonable» no puede concretarse con una regla válida para todos los casos. No obstante, considera que puede alcanzarse una mayor concreción mediante el establecimiento de reglas que establezcan los requisitos con los que tienen que cumplir determinados medios de prueba para constituir prueba de cargo suficiente 
A propósito de umbrales, prototipos y usos de los estándares prueba

Siendo pacífico que el cálculo probabilístico no resulta aplicable para establecer el estándar de prueba en el derecho, sería interesante que Coloma planteara de qué lado de la discusión se encuentra más cercano. Esto es, si considera viable formular un estándar de prueba objetivo e intersubjetivamente controlable que exprese exactamente la ratio de falsos positivos/falsos negativos que estamos dispuestos a aceptar, o más bien, como argumentan Mercedes Fernández, Juan Carlos Bayón y Daniel González, si considera que tal cuestión no resulta viable, por lo que lo que debemos mirar hacia otro lado; concretamente, al momento de conformación del material probatorio y al de la valoración de las pruebas para diseñar reglas legales o jurisprudenciales de prueba que controlen el tipo de pruebas admisibles y los requisitos con los que estas deben cumplir en el momento de su valoración.

No es oportuno desarrollar en este espacio una respuesta al respecto, pero quizá sí resulte útil manifestar mi impresión con la finalidad de suscitar un diálogo con la propuesta de Coloma. Y es que, a mi modo de ver, la discusión se suele plantear en términos dilemáticos: o bien somos capaces de formular un estándar realmente objetivo que exprese con exactitud la distribución del riesgo entre falsos positivos/ falsos negativos, o bien esto no es posible y entonces conviene mirar hacia otro lado. Como he dicho antes, no puedo articular una respuesta completa al respecto en este trabajo, pero me parece que nos encontramos ante un falso dilema.

Como sostiene Susan Haack, se puede admitir que el lenguaje en el que se expresa el estándar de prueba es en parte psicológico, como cuando se habla de «duda» y de «clara y convincente», pero también

para condenar. Por su parte, Juan Carlos Bayón plantea que si el estándar de prueba no es capaz de distribuir adecuadamente el riesgo de error en la determinación de hechos probados habría que intentar hacerlo mediante reglas específicas de prueba. Finalmente, Daniel González Lagier plantea que la formulación de tales reglas debe satisfacer las siguientes condiciones: "(a) Deben tener suficiente flexibilidad como para no incurrir en un nuevo sistema de pruebas rígidamente tasadas"; (b) "deben evitar un lenguaje excesivamente vago e impreciso que, de nuevo, haga imposible el control intersubjetivo de la decisión y (c) deben ser algo más que meros criterios para determinar qué hipótesis está mejor fundada". Vid. Fernández, M., op. cit.; Bayón, J. C., op. cit. y González Lagier, op. cit. 
debemos admitir que el lenguaje en el que se formula el estándar es en parte epistemológico: hablamos de duda razonable y de pruebas claras $y$ convincentes, lo que pone el acento en la calidad de las pruebas, y de manera secundaria en la creencia que se genera con base en las mismas $^{21}$. Tengo la impresión de que en este punto hemos aceptado sin más la tesis de Larry Laudan de descartar por subjetivo cualquier esfuerzo que se ha hecho en la práctica jurisdiccional para analizar el estándar de prueba, cuando la propia práctica ofrece algunos ejemplos de que no todo es subjetivo en el estándar de prueba, ni que todos los intentos que se han realizado son en vano ${ }^{22}$. En contra de lo que plantean Mercedes Fernández, Juan Carlos Bayón y González Lagier se podría argumentar que aún queda mucho por hacer en relación con el estándar de prueba. Por otra parte, trasladar la discusión de la formulación del estándar de prueba al establecimiento de otras reglas sobre la prueba no garantiza sin más que se eviten los problemas que ellos mismos advierten, con lo que se corre el riesgo de estar trasladando simplemente la discusión de un lugar a otro.

(ii) Mi segunda observación tiene que ver con la propuesta de Coloma de entender el estándar de prueba como prototipo. Coloma no establece cuál sería una formulación suficientemente clara del estándar de prueba como prototipo. Ciertamente, pone como ejemplo el estándar de prueba clara y convincente. A su juicio, este estándar indicaría "un tipo de justificación que implica el uso de una narrativa que no abusa en distinciones ni en el lenguaje rebuscado (clara) y, a la vez, que es apta para provocar en el interlocutor adhesión respecto de la estructura argumental utilizada" ${ }^{23}$. Encuentro varios problemas en este planteamiento.

(a) En primer lugar, anteriormente hemos visto que no resulta pacífico por qué habría que entender el estándar de prueba como prototipo.

21 Haack, S., op. cit.

22 Para ilustrar este punto, Susan Haack ofrece varios ejemplos de instrucciones al jurado a nivel estatal y federal en los Estados Unidos en las que se pone el acento en que la atención ha de fijarse en la calidad de las pruebas. Vid. Haack, S., op. cit. 
A propósito de umbrales, prototipos y usos de los estándares prueba

El que la noción de estándar en el lenguaje ordinario pueda entenderse como sinónimo de patrón, modelo o incluso como prototipo no se sigue que el estándar de prueba pueda o deba entenderse también en estos sentidos.

(b) Incluso asumiendo que la noción de estándar como prototipo sea aplicable al estándar de prueba, tengo dudas de que cumpla con la función que le atribuye Coloma. En este sentido, para que sirva como un auténtico patrón o modelo para establecer comparaciones tendríamos que contar con un ejemplar lo suficientemente claro como para evitar los problemas de incertidumbre que detecta Coloma. Esta exigencia no se cumple con la formulación del estándar de prueba clara y convincente que propone Coloma para ilustrar la noción de estándar de prueba como prototipo.

Difícilmente creo que pueda servir como prototipo una formulación del estándar que apele a una narrativa "que no abuse en distinciones" ni tenga "un lenguaje rebuscado" o que genere adhesión por parte del interlocutor. Y principalmente, la propuesta de Coloma resulta problemática porque no remite a las pruebas. A diferencia de la manera habitual en que se entiende el estándar de prueba clara y convincente, Coloma plantea que no son las pruebas las que tienen que ser claras y convincentes, sino la narración empleada por una de las partes.

(c) La propuesta de Coloma de concebir el estándar de prueba como un prototipo me parece problemática por una razón adicional: Coloma apuesta por la teoría de la inferencia a la mejor explicación. Es conveniente advertir que en su aplicación al estándar de prueba, esta teoría tiene el mérito de que pretende reconstruir la manera en que de hecho resuelve el jurado al momento de razonar con hechos. Este es un aspecto que ha sido destacado por Ronald Allen y Ronald Pardo, dos de los principales expositores de esta teoría en Estados Unidos ${ }^{24}$. Como explican estos autores, hay razones para pensar que el jurado de hecho razona y formula su veredicto en función de aquellas reconstrucciones que les parezcan mejores o más sólidas o razonables. Sin embargo, el

${ }^{24}$ Allen, R. op. cit. y Pardo, M., op. cit. 
que esto sea así en los Estados Unidos no debe llevarnos a pensar que así razonen o así deban razonar los tribunales en nuestro contexto.

Distintos autores han señalado que la teoría de la inferencia a la mejor explicación no es un buen candidato para formular el estándar de prueba. Entre ellos, Juan Carlos Bayón ha planteado, retomando la crítica de Larry Laudan a esta teoría, que el criterio de "mejor explicación" no es un buen candidato para adoptarlo como criterio de decisión $^{25}$. A pesar de que sea la "mejor" explicación puede que no resulte adecuada para basar la decisión de considerar probado un hecho en el campo del derecho. Y eso al menos por dos razones: puede tratarse de la mejor o la menos mala de dos explicaciones insuficientemente plausibles y por tanto inadecuadas para considerar probada la hipótesis en la que se basa dicha explicación. Y en segundo lugar, es posible que la peor de dos explicaciones sea lo suficientemente plausible como para considerar probada la hipótesis a la que dicha explicación se refiere. Pero la inferencia a la mejor explicación no resulta adecuada por una razón aún más fundamental. Como señala Juan Carlos Bayón: "la inferencia a la mejor explicación no pretende ser un estándar de prueba: no pretende distribuir el riesgo del error, sino atribuir racionalmente grados de confirmación a las distintas hipótesis" 26 . En otras palabras, la inferencia a la mejor explicación resulta útil en la etapa de valoración de las pruebas para determinar qué hipótesis prevalece sobre otra, pero no en el momento de toma de decisión con base en el estándar de prueba, o al menos no si pretendemos que el estándar de prueba tenga una sensibilidad más que mínima frente al riesgo de error $^{27}$.

${ }^{25}$ Bayón, op. cit. y Laudan, L., "Aliados extraños. La inferencia a la mejor explicación y el estándar de prueba", trad. Edgar Aguilera, Problema. Anuario de Filosofia y Teoría del Derecho, 2007, 1, pp. 305-327.

26 Bayón, J. C., op. cit.

${ }^{27}$ Como señala González Lagier, también habría que tomar en cuenta además que la formulación del estándar que hacen autores como Allen y Pardo contiene expresiones cargadas de vaguedad. González Lagier, op. cit., p. 114. 
A propósito de umbrales, prototipos y usos de los estándares prueba

\section{Sobre los usos y macro-usos de los estándares de prueba}

El cuarto y último apartado del artículo de Coloma está dedicado al tema de los usos de los estándares de prueba. Como se anticipó al comienzo de este trabajo, el propósito que persigue Coloma en este punto es plantear que el estándar de prueba cumple varias funciones adicionales a la de distribuir el error entre las partes en el proceso. Coloma identifica seis usos generales de los estándares de prueba que posteriormente agrupa en los tres macro-usos siguientes: i) determinación de la cantidad de errores esperables de sistema de adjudicación, ii) distribución de los errores entre las partes y iii) forma que adoptan los hechos probados.

A continuación, formularé algunos comentarios sobre alguno de los usos y macro-usos que identifica Coloma.

Concuerdo en términos generales con la tesis de Coloma. Intuitivamente, se puede aceptar por ejemplo que el estándar de prueba puede servir para orientar a las partes de la conveniencia de llevar o no un caso a juicio dependiendo de su nivel de exigencia o que la mayor o menor exigencia del estándar de prueba puede influir en los costos que para las partes supone tener que probar ciertas pretensiones o acusaciones. Este tipo de afirmaciones resultan plausibles a nivel general, pero si se pretende que tengan un alcance mayor hace falta algún sustento empírico de lo que ocurre de hecho en tal o cual práctica jurídica.

Hay otros usos de los estándares de prueba que me parecen más problemáticos y sobre los que me limitaré a formular algunos comentarios puntuales. En este sentido, Coloma sostiene que los estándares de prueba "modelan la clase de inferencias que es válido realizar". Desde esta perspectiva, señala que los estándares de prueba "definen la magnitud de saltos argumentales que resulten tolerables", entendiendo por "saltos argumentales" "aquellos que van mucho más allá de lo que sus premisas permiten" 28 . No me queda muy claro que este sea propiamente un uso de los estándares de prueba. En caso de que pudiera entenderse efectivamente como un uso, me parece que el estándar no 
estaría cumpliendo con su principal cometido: establecer las condiciones para determinar que un hecho se puede considerar probado. No veo de qué modo un estándar de prueba pudiera incorporar entre sus requisitos para la toma de decisión de un hecho como probado el que no incurra en saltos argumentales. En caso de que se aceptara, se trataría de un requisito para la solidez de la inferencia probatoria, pero no de un requisito para la toma de decisión. El hecho de que haya un salto argumental en alguno de los tramos de la inferencia convertiría a esta en inaceptable.

Por otra parte, Coloma señala que otro de los usos de los estándares de prueba es que "influyen en la clase de razonamiento que las partes y los jueces adoptan”, señalando que habría dos enfoques en competencia: el enfoque atomista y el enfoque holista ${ }^{29}$. El primer enfoque consistiría en distinguir y analizar separadamente las distintas proposiciones clasificándolas en distintos niveles dependiendo de que se traten de hipótesis referidas a los hechos principales o secundarios. Desde este primer enfoque, el razonamiento probatorio se presenta como una cadena de inferencias que va de las pruebas a cada uno de los hechos últimos a probar ${ }^{30}$.

El segundo enfoque concibe el razonamiento probatorio como un todo unitario en el que las eventuales deficiencias o virtudes al interior del razonamiento han de ser examinadas en conjunto y no de manera aislada. Al respecto, mi comentario es similar al anterior. No veo de qué manera la elección por un enfoque atomista u holista puede considerarse como un uso de los estándares de prueba. Por otra parte, al plantear la cuestión como un enfoque atomista u holista se corre el riesgo de que se esté mandando el mensaje de que se trata de opciones igualmente disponibles para el órgano decisor, cuando habría que hacer hincapié en que debe imperar un enfoque atomista y analítico

29 Vid. Coloma, op. cit.

30 Para un ejemplo paradigmático de este enfoque, vid. Anderson, T., Twining, W. y Schum, D., Analysis of Evidence, segunda edición, 2005, Cambridge University Press. Trad. en castellano: Análisis de la prueba, trad. coordinada por Flavia Carbonell y Claudio Agüero, Marcial Pons, 2015. 
A propósito de umbrales, prototipos y usos de los estándares prueba

del razonamiento probatorio y que el enfoque holista podría tener en todo caso un papel complementario en la valoración de las pruebas ${ }^{31}$.

Finalmente, en el primero de los macro-usos que distingue Coloma señala que los estándares de prueba influyen en la "determinación de la cantidad de errores esperables del sistema de adjudicación" 32 . Coloma afirma en este sentido que "un estándar de prueba exigente favorece que las decisiones adoptadas por los tribunales de justicia sean, en términos generales, más fiables que las que resultan de la utilización

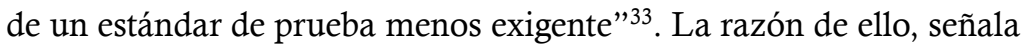
Coloma, es que "con un EdP exigente la parte demandante o acusadora elige solo «buenos casos»; es decir, aquellos en los que vislumbra que tiene posibilidades de éxito" 34 . Al respecto, no alcanzo a ver la conexión que plantea Coloma entre el nivel de exigencia del estándar de prueba y el grado de fiabilidad de la decisión. Las partes pueden tener distintas razones para considerar que tienen un «buen caso», sin que ello suponga que haya más probabilidad de que se produzca una decisión más fiable. En contra de lo que afirma Coloma, el estándar de prueba no incide propiamente en la mayor o menor fiabilidad de la decisión (esta sería más bien la función de los criterios de valoración racional de la prueba) sino en la manera en que se asigna y se distribuye el error entre las partes.

\section{Bibliografía}

Accatino, D., "Atomismo y holismo en la justificación probatoria", Isonomía, 40, 2014, pp. 17-59.

Accatino, D. "Certezas, dudas y propuestas en torno al estándar de la prueba penal”, Revista de Derecho de la Pontífica Universidad Católica de Valparaíso, 2011, pp. 483-511.

31 Vid. en este sentido Accatino, D., "Atomismo y holismo en la justificación probatoria", Isonomía, 40, 2014, pp. 17-59.

32 Vid. Coloma, op. cit.

33 Vid. Coloma, op. cit.

34 Vid. Coloma, op. cit. 
Allen, R. J., "Burdens of proof", Law, Probability and Risk, 2015, 3 (3-4), pp. 195-219.

Anderson, T., Twining, W. y Schum, D., Analysis of Evidence, segunda edición, 2005, Cambridge University Press. Trad. en castellano: Análisis de la prueba, trad. coordinada por Flavia Carbonell y Claudio Agüero, Marcial Pons, 2015.

Bayón, J. C., "Epistemología, moral y prueba de los Hechos: hacia un enfoque no benthamiano", Analisi e Diritto, 2008, pp. 15-34.

Diccionario Anaya de la Lengua, Madrid, Anaya, 1991.

Diccionario de la Lengua Española, España, La Academia, 2001.

Fernández, M., "La valoración de la prueba y el estándar de duda razonable", Cuadernos Electrónicos de Filosofia del Derecho, 2007, 15.

Ferrer, J., La valoración racional de la prueba, Madrid-Barcelona: Marcial Pons, 2005.

Gascón, M., "Sobre la posibilidad de formular estándares de prueba objetivos", Doxa, 28, 2005, pp. 127-139

González Lagier, D., "Presunción de inocencia, verdad y objetividad", en García Amado, J. A. y Bonorino, P (coords.), Prueba y razonamiento probatorio, Granada, 2014, pp. 85-117.

Haack, S., "El probabilismo jurídico. Una disensión epistemológica", en Vázquez, C. (ed.), Estándares de prueba y prueba científica. Ensayos de epistemología jurídica, Madrid: Marcial Pons, 2013, pp. 65 y ss.

Haack, S., Evidence matters. Science, Proof and Truth in the Law, New York: Cambridge, 2014.

Kaplow, L. "Rules versus standards: an economic analysis", Duke Law Journal, 43, 1992.

Igartua, J., "Prolongaciones a partir de Laudan", Doxa, 28, 2005, pp. 1412-1150.

Fernández, M., "La valoración de la prueba y el estándar de duda razonable", Cuadernos Electrónicos de Filosofia del Derecho, 2005.

Igartua, J., "Tomando en serio la duda razonable: enseñanzas de un controvertido caso judicial", en Derecho procesal: dilemas sobre la verdad en el proceso judicial, Medellín: Editorial Universidad Pontificia Bolivariana, 2014, pp. 135-162. 
Laudan, L., "Por qué un estándar de prueba subjetivo y ambiguo no es un estándar", Doxa, 28, 2005, pp. 95-13.

Laudan, L., "Aliados extraños. La inferencia a la mejor explicación y el estándar de prueba", trad. Edgar Aguilera, Problema. Anuario de Filosofia y Teoría del Derecho, 2007, 1, pp. 305-327.

Laudan, L., Verdad, errory proceso penal, trad. Carmen Vázquez y Edgar Aguilera, Marcial Pons, 2013.

Schauer, F., "The convergence of rules and standards", New Zealand Law Review, 303, 2003. 